\title{
CIENCIA\&SALDD
}

\section{ACTUALIZACIÓN EN DIAGNÓSTICO Y TRATAMIENTO DE}

HIPERHIDROSIS LOCALIZADA PRIMARIA

Juliana Salazar Mayorga' Guido Angulo Ureña² Alberto Quesada Pacheco ${ }^{3}$

1 y 3 Médico general. Trabajador independiente, San José Costa Rica.

2 Médico general. Hospital CIMA, San José. Costa Rica.

Contacto: Julianasm93@gmail.com guidono123@gmail.com betoqp@gmail.com

\section{RESUMEN}

La hiperhidrosis localizada primaria es una condición dermatológica caracterizada por una producción excesiva de sudor que supera las necesidades termoreguladoras, afectado zonas localizadas como palmas, plantas, axilas o región craneofacial. Su etiología sigue siendo incierta, aunque la evidencia sugiere un componente genético importante. El diagnóstico requiere de una historia clínica exahustiva que permita descartar causas secundarias y farmacológicas. Algunas pruebas cualitativas y cuantitativas pueden ser de utilidad en el diagnóstico. Dada la importante afectación en la calidad de vida de los pacientes, existen diferentes opciones terapéuticas disponibles desde agentes tópicos y dispositivos médicos, hasta terapias sistémicas e intervenciones quirúrgicas. Estos varían mucho en cuanto a efectividad, seguridad, tolerabilidad y costo, por lo que se debe individualizar la terapia, tomando en consideración la afectación en la calidad de vida, la localización de la enfermedad, las contraindicaciones y las posibles complicaciones.

Palabras clave: Hiperhidrosis, sudoración, cloruro de aluminio, toxinas botulínicas, iontoforesis, simpatectomía.

\section{Cómo citar:}

Salazar Mayorga, J., Angulo Ureña, G., \& Quesada Pacheco, A. (2021). Actualización en diagnóstico y tratamiento de hiperhidrosis localizada primaria. Revista Ciencia Y Salud, 5(1), Pág. 59-68.

Recibido: 16/dic/2020 Aceptado: 18/ene/2021 Publicado: 15/feb/2021

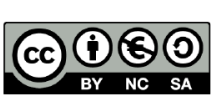

\section{ABSTRACT}

Primary or focal hyperhidrosis is a dermatologic condition characterized by excessive sweat production surpassing the thermoregulation needs, affecting focal zones like palms, soles, axillae, or craneofacial region. Its etiology remains uncertain, although evidence suggests there is an important genetic component. The diagnosis requires an exhaustive clinical history that allows the clinician to rule out secondary and pharmacological causes. Some qualitative and quantitative tests may be useful in the diagnosis. Given the important impact on patient's life quality, there are different therapeutical options available; from topical agents and medical devices, to systemic therapies and surgical procedures. These vary a lot in terms of effectiveness, safety, tolerability, and cost, and thus therapy choice must be individualized, taking into account the quality of life affected, the location of the disease, contraindications, and possible complications.

Keywords: Hyperhidrosis, sweating, aluminum chloride, botulinum toxins, iontophoresis, sympathectomy. 


\section{CIENCIA\&SALUD}

\section{INTRODUCCIÓN}

La hiperhidrosis es una condición dermatológica caracterizada por sudoración excesiva en cantidades superiores a las necesarias fisiológicamente para la termorregulación. Puede clasificarse según su etiología en primaria o secundaria, esta última originada por trastornos neurológicos, endocrinos, infecciosos, tumorales o por algunos fármacos. También puede clasificarse en función de su localización en generalizada o localizada.

La hiperhidrosis localizada puede ser primaria, la cual se manifiesta a nivel axilar, palmar, plantar o craneofacial; o secundaria que en general es asimétrica y de origen neurológico. La hiperhidrosis suele ser incontrolable y puede conllevar a trastornos físicos como infecciones cutáneas o psicológicos como depresión o ansiedad y a una importante afectación en la calidad de vida, por lo que el criterio más importante para decidir el tipo de terapia consiste en la afectación individual de cada paciente en su calidad de vida.

Las opciones terapéuticas varían de acuerdo con su localización focal o generalizada e incluye desde la terapia tópica u oral, hasta el manejo quirúrgico.

\section{MÉTODOS}

Se realizó una revisión bibliográfica extensiva en las bases de datos de PubMed y ScienceDirect, eligiendo bibliografía acorde con el tema. Se dio un énfasis en artículos más actualizados y aquellos enfocados en tratamientos emergentes. Se eligieron 14 artículos en total con este fin.

No existen conflictos de interés a declarar.

\section{EPIDEMIOLOGÍA}

Los estudios de prevalencia de la enfermedad varían ampliamente según la población y los métodos de estudio, la mayoría han analizado a una porción limitada de la población por lo que sus conclusiones no pueden generalizarse. La prevalencia poblacional más citada estima un $2.8 \%$ de acuerdo con una encuesta estadounidense realizada en el 2004 en 150.000 hogares, sin embargo, esta encuesta no contemplaba el sitio de sudoración excesiva (1).

Una encuesta del 2016 sugirió que aproximadamente el 4,8\% de la población estadounidense o 15,3 millones de personas tienen hiperhidrosis, sin embargo, se especula que esta es una subestimación ya que esta prevalencia está muy por debajo de lo reportado en otras poblaciones.

Algunos factores culturales y sociales pueden afectar la percepción o disposición de los pacientes para reportar la sudoración anormal, y en aquellos con síntomas leves resulta menos probable que notifiquen los síntomas en comparación con casos severos.

Una revisión de 2011 de Nawrocki, et. al indicó que el 93\% de los pacientes con hiperhidrosis presentan hiperhidrosis primaria localizada (2).

En un estudio retrospectivo en Brasil se analizaron las diferentes presentaciones de hiperhidrosis en 1278 pacientes que acudieron a una clínica especializada durante un período de 7 años, y se concluyó que la hiperhidrosis se manifiesta con frecuencia en más de un sitio, siendo la localización palmar la principal queja de los pacientes. Cuando se presentan dos sitios de sudoración la combinación más frecuente es palmar + axilar y cuando hay tres sitios de sudoración la combinación más frecuente es palmar + plantar + axilar (3).

Tanto los hombres como las mujeres se ven afectados por igual, aunque las mujeres buscan tratamiento con mayor frecuencia que los hombres.

El inicio de la hiperhidrosis primaria es más común entre los 14 y los 25 años. Cuando se manifiesta en pre- 


\section{CIENCIA\&SALUD}

púberes generalmente es palmar o plantar (88.9\%), con presentaciones a nivel axilar (15.5\%) o facial (6.6\%) menos comunes. El inicio pospuberal se asocia más frecuentemente con una distribución axilar (4).

Según Henning, et. al existen antecedentes familiares positivos en 35-56\% de los casos de hiperhidrosis primaria y la herencia parece ser autosómica dominante. Los individuos con fenotipos palmoplantares y antecedentes familiares positivos suelen tener una edad de inicio más temprana. Se han encontrado dos locus para hiperhidrosis en los cromosomas 2 q31.1 y 14q11.2-q13, sin embargo, la evidencia es heterogénea y limitada y parece ser que la hiperhidrosis se hereda poligenéticamente (5).

\section{ETIOLOGÍA}

La termorregulación involucra la disipación del calor corporal mediante la evaporación del sudor. Existen tres tipos de glándulas sudoríparas: ecrinas, apocrinas y apoecrinas. Las ecrinas son las responsables mayoritariamente de la hiperhidrosis primaria. Estas son particularmente abundantes en palmas, plantas, frente, axilas y mejillas, y son las únicas glándulas presentes en las palmas. Están ausentes en mucosa labial, conducto auditivo externo, lecho ungueal y genitales donde predominan las glándulas apocrinas.

Las glándulas ecrinas producen una secreción fina, hipotónica, transparente e inodora, y la tasa y volumen de producción fluctúa de acuerdo con las necesidades termoreguladoras.

Estas glándulas ecrinas están inervadas por fibras colinérgicas. La acetilcolina, secretada por las terminaciones nerviosas, estimula la producción de sudor.

La tasa de secreción normal de las glándulas sudoríparas ecrinas es de 0.5 a $1 \mathrm{~mL} / \mathrm{minuto}$ y solo el $5 \%$ de ellas secretan sudor en cualquier momento. En la hiperhidrosis grave, la secreción puede exceder los 40 $\mathrm{mL} / \mathrm{m} 2 /$ minuto.

La producción de sudor está regulada por estructuras de la corteza cerebral, la región preóptica anterior del hipotálamo y el sistema nervioso simpático. Las fibras nerviosas aferentes llevan señales al hipotálamo a través de la médula espinal lateral y la vía eferente simpática sigue el trayecto desde la corteza cerebral hasta el hipotálamo, luego a la médula, donde las fibras cruzan el bulbo raquídeo y continúan hasta el cuerno lateral de la médula espinal, de ahí pasan a los núcleos intermediolaterales de los ganglios paravertebrales simpáticos y las fibras nerviosas simpáticas tipo C posganglionares estimulan los receptores muscarínicos postsinápticos de la glándula sudorípara ecrina (2).

En una revisión sistemática de Hasmonai sobre la etiología de la hiperhidrosis se determinó que existen posibles diferencias histoanatómicas y fisiológicas, así como posibles disfunciones metabólico-enzimáticas y neurológicas en los pacientes con hiperhidrosis en comparación con personas con transpiración normal. Estas diferencias se pueden clasificar en tres grandes grupos:

A) Un rasgo genético hereditario identificado en los cromosomas 2 y 14.

B) Un mayor número y tamaño de células ganglionares y una vaina de mielina más gruesa de los axones involucrados.

C) Una mayor expresión de subunidades del receptor de acetilcolina en los ganglios simpáticos de sujetos con hiperhidrosis (6).

\section{DIAGNÓSTICO}

Las causas de hiperhidrosis secundaria deben ser excluidas antes de poder diagnosticar una hiperhidrosis primaria. En la historia clínica se deben abordar las interrogantes sobre el patrón de sudoración, edad de inicio, factores agravantes, duración, frecuencia, cantidad, distribución, sudoración nocturna, historia familiar y síntomas que orienten hacia una causa secundaria como uso de medicamentos, pérdida de peso, fiebre o linfadenopatías. 


\section{CIENCIA\&SALUD}

El examen físico se debe enfocar en valorar la sudoración excesiva, la cual puede evidenciarse de manera cuantitativa o cualitativa mediante algunas pruebas o cuestionarios diagnósticos (ver anexo 1),

Los exámenes de laboratorio usualmente no son necesarios para el diagnóstico de hiperhidrosis primaria localizada, sin embargo pueden ser de utilidad en la hiperhidrosis secundaria (2).

Los criterios diagnósticos específicos para hiperhidrosis primaria localizada incluyen:

- Sudoración excesiva en al menos uno de los siguientes sitios: axilas, palmas, plantas o región craneofacial.

- Duración superior a seis meses.

- Descartar posibles causas secundarias.

- $\quad$ Presentar al menos dos de las siguientes características:

- Sudoración bilateral y relativamente simétrica

- $\quad$ Edad de aparición por debajo de los 25 años.

- $\quad$ Antecedentes familiares positivos por hiperhidrosis focal primaria.

o $\quad$ No hipersudoración durante el sueño.

- $\quad$ Repercusión sobre las actividades de la vida diaria (7).

Los métodos cuantitativos y cualitativos para examinar la producción de sudor no se realizan comúnmente durante el examen clínico, sin embargo, pueden ayudar a determinar la gravedad de la enfermedad, a orientar el tratamiento y a recopilar datos para la investigación clínica.

La prueba de Minor con almidón y yodo estima una aproximación cualitativa del volumen de sudor producido mediante un mapa de las regiones afectadas por sudoración antes del inicio del tratamiento. Esta se realiza aplicando polvo de maicena y yodo en las superficies afectadas, estos componentes reaccionan con el sudor y producen un sedimento violáceo que identifica las áreas afectadas.

La prueba de ninhidrina implica su reacción con aminoácidos presentes en el sudor produciendo colores vivos que se pueden cuantificar a través de análisis digital.

Las pruebas gravimétricas permiten pesar el sudor producido en un área específica durante un período de tiempo, utilizando papel de filtro previamente pesado.

Existe también la prueba termoreguladora en la cual se aplica rojo de alizarina, almidón de maíz y carbonato de sodio para identificar áreas hiperhidróticas. Posteriormente se coloca al paciente en una cámara calentada, lo que permite diferenciar la sudoración termorreguladora de la hiperhidrótica.

La sudorimetría cuantitativa dinámica mide la sudoración a través del tiempo mediante una cámara colocada en la superficie de la piel por la que se transmite gas seco.

El medidor de humedad electrónico se utiliza para detectar la evaporación de la humedad de la piel directamente.

Por otro lado, dentro de los métodos cualitativos para valorar la hiperhidrosis, existe la Escala de severidad de la hiperhidrosis (HDSS), que es un cuestionario por medio del cual los pacientes clasifican la afectación del sudor en sus actividades cotidianas mediante una escala de 4 grados. Un puntaje 3-4 indica enfermedad severa, 2 indica enfermedad moderada y 1 indica ausencia de hiperhidrosis. Esta escala también permite valorar el éxito del tratamiento definido por un cambio en el puntaje de 4 o 3 a 2 o 1, cada punto ha demostrado una correlación de un 50\% de reducción en la sudoración.

Otro cuestionario utilizado en la práctica clínica es el Cuestionario de impacto de la hiperhidrosis, que consiste en 41 ítems basales y 10 ítems para el seguimiento y permite valorar tanto el impacto en la vida cotidiana como la eficacia del tratamiento. 


\section{CIENCIA\&SALUD}

El Índice de calidad de vida en dermatología (Dermatology Life Quality Index, DLQI) es otra herramienta utilizada en varias condiciones dermatológicas que permite valorar tanto el efecto como la mejoría de la calidad de vida de los pacientes (2).

\section{DIAGNÓSTICOS DIFERENCIALES}

Para el diagnóstico diferencial de hiperhidrosis primaria localizada, siempre es importante considerar y descartar todas las posibles causas de hiperhidrosis secundaria.

La mayoría de las veces la hiperhidrosis secundaria suele presentarse de forma generalizada, la cual ocurre tanto en sueño como en vigilia y puede ser causada por condiciones fisiológicas como calor excesivo, fiebre, embarazo o menopausia. Las causas patológicas incluyen malignidad (linfoma, trastornos mieloproliferativos), infecciones (viral, bacteriana, tuberculosis, malaria, VIH), trastornos metabólicos o endocrinológicos (diabetes, hipertiroidismo, feocromocitoma, acromegalia, hiperpituitarismo o síndrome carcinoide), enfermedad cardiovascular (endocarditis, falla cardíaca congestiva), o enfermedades neurológicas (ictus o Parkinson), trastornos psiquiátricos entre otros (2).

Algunas drogas que podrían asociarse a hiperhidrosis secundaria generalizada son: antidepresivos, ansiolíticos, antipsicóticos, agonistas colinérgicos, agentes hipoglicemiantes, moduladores selectivos de receptores de estrógenos, triptanes, antipiréticos, AINES, antieméticos, alcohol, cocaína, heroína, entre otros (8). La hiperhidrosis secundaria localizada puede ser simétrica asociada a hiperhidrosis compensatoria por la simpatectomía o sudoración gustatoria que cuando es fisiológica se asocia a comidas calientes o picantes, pero puede ser patológica posterior a simpatectomía, paroidectomía o postinfección por herpes zóster. Cuando se trata de hiperhidrosis secundaria localizada asimétrica se puede deber a lesiones o tumores neurológicos como nevus ecrino o síndrome de Frey (2).

\section{TRATAMIENTO}

Dependiendo de la localización de la hiperhidrosis, existen diferentes opciones terapéuticas. El tratamiento debe ser individualizado considerando las contraindicaciones y la posibilidad de falla terapéutica (9).

Las opciones de tratamiento incluyen agentes tópicos, orales, inyectables, así como dispositivos médicos y opciones quirúrgicas que varían mucho en cuanto a efectividad, seguridad, tolerabilidad y costo (10).

\section{TERAPIA TÓPICA}

Antitranspirantes tópicos:

El tratamiento de primera línea para la hiperhidrosis focal primaria, independientemente de la gravedad es el cloruro de aluminio tópico (8).

Se utilizan formulaciones de $20 \%$ de cloruro de aluminio en alcohol etílico, $6.25 \%$ de tetracloruro de aluminio y $12 \%$ de cloruro de aluminio en agua carbonatada. Las sales de aluminio provocan obstrucción de las glándulas sudoríparas ecrinas y destrucción de las células secretoras.

Para obtener resultados óptimos, la solución debe ser aplicada durante las noches cada 24-48 horas y debe permanecer en una piel de 6-8 horas antes de lavar. Una vez que se logra la anhidrosis, el intervalo de aplicación debe ser de 1-2 veces por semana.

Cuatro estudios observacionales han demostrado la seguridad y eficacia del cloruro de aluminio para el control de la hiperhidrosis. 


\section{CIENCIA\&SALUD}

A pesar de los resultados satisfactorios, gran cantidad de pacientes experimentan cierto grado de irritación cutánea, especialmente cuando se aplica sobre la piel húmeda al convertirse en ácido clorhídrico, este constituye el principal motivo de interrupción del tratamiento, el cual puede limitarse aplicándose sobre la piel seca, aumentando los intervalos de aplicación o utilizando corticosteroides de baja potencia.

Se ha demostrado que la preaplicación de vaselina mejora la función de barrera y previene los efectos irritantes (10).

Otras sales utilizadas con menos frecuencia incluyen el circonio, vanadio e indio las cuales se cree que funcionan a través del mismo mecanismo (11).

Existen controversias sobre el impacto que tiene la exposición al aluminio y el riesgo de cáncer de mama. Según la Agence Francaise de Sécurité Sanitaire des Produits de Santé (AFSSAPS), el National Cancer Institute $(\mathrm{NCl})$ y algunos estudios, no existe una relación formal entre la utilización de productos cosméticos que contengan sales de aluminio y el cáncer de mama (12).

Anticolinérgicos tópicos:

El glicopirrolato (solución, gel o crema al 0.5-4\%) y la oxibutinina tópica al 3\% son los agentes anticolinérgicos tópicos más comúnmente utilizados. Sus efectos adversos incluyen: irritación cutánea, prurito, cefalea, mareos, odinofagia, midriasis, boca seca, estreñimiento y nasofaringitis (11).

\section{TERAPIA INYECTABLE}

Toxina botulínica Tipo A (BTX A)

Las inyecciones locales intradérmicas con BTX A constituyen un tratamiento eficaz. Estudios multicéntricos aleatorios han demostrado mayor utilidad en hiperhidrosis axilar.

La BTX A provoca una denervación química local previniendo la liberación de acetilcolina. La duración del efecto varía entre individuos y el tratamiento generalmente debe repetirse 1-4 veces al año (9).

La mayoría de estudios han utilizado una de las dos preparaciones de BTX A: onabotulinum (Botox), abobotulinum (Dysport) o incobotulinum (Xeomin) (11). Las primeras dos preparaciones tienen diferentes dosis; 1 unidad de Botox equivale aproximadamente a 3 unidades de Dysport (9).

Toxina botulínica tipo B (BTX B)

El mecanismo de acción de la BTX B es similar al de la BTX A, pero el efecto sobre las motoneuronas alfa sobre el músculo parece ser menor, ya que se requieren dosis 50-100 veces más altas para tratar la distonía cervical.

Sin embargo, se ha demostrado que se puede diluir la BTX B a una concentración baja para que el efecto inhibitorio de $1 \cup$ de BTX A corresponda a 1-2 U de BTX B (9).

La duración del efecto con BTX B suele ser más corta ( 3 meses) en comparación con BTX A, pero con un período de respuesta más corto (5-7 días) (11).

Los efectos no deseados más comunes incluyen el dolor en el sitio de inyección, malestar, irritación, inflamación o hematomas. El dolor es más intenso con BTX B en comparación con BTX A probablemente debido a su pH más ácido.

El dolor es un factor importante que limita la utilidad de la toxina botulínica. Algunos métodos comunes 


\section{CIENCIA\&SALUD}

para el alivio del dolor incluyen: una técnica de inyección adecuada con analgesia local, crioanestesia, anestésicos tópicos, dilución de la toxina en lidocaína, bloqueo de nervios ulnar, mediano y radial (10).

\section{DISPOSITIVOS MÉDICOS}

Iontoforesis

La iontoforesis puede ser una alternativa segura y costo-efectiva aprobada por la FDA para el tratamiento la hiperhidrosis palmo-plantar.

Utiliza una corriente galvánica que se transmite a través de la piel intacta sumergida en un medio líquido. El electrodo cargado positivamente repele los hidrogeniones de los ductos ecrinos (11).

El mecanismo de acción exacto aún es incierto, pero algunas hipótesis sugieren la inhibición de la transmisión simpática, la obstrucción de las glándulas sudoríparas mediante depósitos de iones y la alteración del $\mathrm{pH}$ local que inhibe a las glándulas sudoríparas (13).

Se utiliza 3-4 veces por semana de 20-30 minutos a una corriente de 15-20 mA y se requieren aproximadamente de 6-15 sesiones para alcanzar la anhidrosis, el efecto permanece de 2-14 semanas y es efectiva en un $80 \%$ de los casos.

Algunos estudios sugieren que las modificaciones del medio acuoso con cloruro de aluminio, agentes anticolinérgicos o toxina botulínica, pueden aumentar el efecto anhidrótico. Los efectos adversos más frecuentes incluyen: disconformidad, sequedad, parestesias, eritema o vesiculación de las áreas afectadas (11).

\section{OTROS DISPOSITIVOS MÉDICOS}

El ultrasonido, la termólisis por microondas, el láser y la radiofrecuencia pueden considerarse para el tratamiento de hiperhidrosis axilar, sin embargo, se debe considerar su alto costo, y la destrucción potencialmente permanente e irreversible, los datos clínicos aún son limitados y dependen de la experiencia del médico tratante.

La termólisis por microondas (Miradry) ha sido aprobada por la FDA para hiperhidrosis axilar. Esta genera una destrucción de las glándulas ecrinas por rotación de moléculas de agua. Tiene una eficacia del 90\% que persiste por más de 12 meses.

La terapia con ultrasonido proporciona bajos niveles de lesión térmica focalizada en las unidades ecrinas con un $80 \%$ de reducción de la sudoración y ha sido aprobada por la FDA para procedimientos estéticos de estiramiento de la piel.

La radiofrecuencia fraccional involucra la inserción de microagujas en la piel que emiten energía termal bipolar directamente sobre las glándulas ecrinas con mínimo trauma epidérmico (11).

La eficacia de los tratamientos con láser para la hiperhidrosis primaria es controversial. En varios estudios, se ha utilizado el láser Nd:YAG con parámetros similares a los utilizados para la depila-ción, logrando una disminución subjetiva de la sudoración. Sin embargo, la falta de estudios clínicos que permitan establecer un protocolo de tratamiento, especialmente en la población pediátrica, ha impedido que sea un tratamiento recomendado (14).

\section{TERAPIA ORAL}

Los principales agentes sistémicos usados para tratar la hiperhidrosis focal son los anticolinérgicos, debido 


\section{CIENCIA\&SALUD}

a que inhiben la señalización de acetilcolina a nivel neuroglandular. Entre los medicamentos orales disponibles para el tratamiento de la hiperhidrosis, se cuenta actualmente con agentes anticolinérgicos (oxibutinina, glicopirrolato, bromuro de mentatelina) y los agonistas alfa-adrenérgicos (clonidina). La principal limitación de estos medicamentos es la gran frecuencia de efectos secundarios como boca seca, visión borrosa, retención urinaria, estreñimiento y taquicardia por lo que se prefiere su uso en la hiperhidrosis generalizada (14).

\section{MANEJO QUIRÚRGICO}

La cirugía se reserva como última línea de tratamiento, siendo utilizada luego del fracaso con otras intervenciones menos invasivas.

Existen algunas técnicas quirúrgicas locales que han sido utilizadas en el manejo de la hiperhidrosis axilar. Estas técnicas incluyen la escisión radical quirúrgica, liposucción y el curetaje que, aunque inicialmente han demostrado reducir la sudoración axilar, tienen altas tasas de recidiva (8).

La simpatectomía torácica endoscópica se utiliza en casos graves y refractarios al tratamiento.

Implica el uso de toracoscopia asistida por video para interrumpir una sección de la cadena ganglionar simpática mediante, transección, ablación, o clampeo de los ganglios torácicos involucrados (T3 o T4), evitando que las señales nerviosas lleguen a las glándulas sudoríparas (10).

Aunque el procedimiento disminuye o elimina de forma permanente la sudoración en el área original, una complicación tardía común es la sudoración compensatoria en otras áreas generalmente abdomen, espalda, región glútea y piernas (8).

\section{CONCLUSIONES}

La hiperhidrosis primaria localizada es una enfermedad con gran impacto social y psicológico. Existe una alta prevalencia de antecedentes familiares positivos y se cree que se basa en un mayor número y tamaño de células ganglionares simpáticas y una mayor expresión de subunidades del receptor de acetilcolina en los ganglios simpáticos. Es de especial importancia la historia clínica para poder descartar posibles causas secundarias sistémicas o farmacológicas y definir el cumplimiento de los criterios diagnósticos.

Algunas pruebas de utilidad diagnóstica que permiten valorar de forma cualitativa y cuantitativa la hiperhidrosis son la prueba de Minor (utilizada con mayor frecuencia), prueba de ninhidrina, pruebas gravimétricas y termoreguladoras, así como ciertas escalas como el DLQI o la HDSS, que permiten valorar tanto el efecto de la terapia, como la mejoría en la calidad de vida de los pacientes.

El tratamiento tópico como los antitranspirantes a base de cloruro de aluminio corresponde a la primera línea de tratamiento. Otras terapias como la toxina botulínica, la iontoforesis, la termólisis por microondas o los tratamientos sistémicos están indicados para casos moderado-severos cuando la terapia tópica es insuficiente o está contraindicada. El manejo quirúrgico mediante la simpatectomía torácica endoscópica se reserva como última línea de tratamiento para casos graves, refractarios a otras terapias, tomando en consideración las posibles complicaciones como la sudoración compensatoria. 


\title{
CIENCIA\&SALUD
}

\section{ANEXOS}

\author{
Localised excessive sweating of at least 6 months duration with \\ unknown cause with minimum of 2 of following traits \\ Bilateral and symmetric sweating \\ Occurs at least once/week \\ Impairs daily activities \\ Age at onset $<25$ years \\ Positive family history \\ Sweating ceases during sleep
}

Anexo 1. Criterios diagnósticos para hiperhidrosis focal primaria.

\section{REFERENCIAS BIBLIOGRÁFICAS}

1. Ricchetti-Masterson, Kristen, et al. "Epidemiology of Hyperhidrosis in 2 Population-Based Health Care Databases." Journal of the American Academy of Dermatology, vol. 78, no. 2, 2018, pp. 358-362., doi:10.1016/j.jaad.2017.10.004.

2. Nawrocki, Shiri, and Jisun Cha. "The Etiology, Diagnosis, and Management of Hyperhidrosis: A Comprehensive Review." Journal of the American Academy of Dermatology, vol. 81, no. 3, 2019, pp. 657-666., doi:10.1016/j.jaad.2018.12.071.

3. Estevan, Fernanda Alvarenga, et al. "Epidemiologic Analysis of Prevalence of the Hyperhidrosis." Anais Brasileiros De Dermatologia, vol. 92, no. 5, 2017, pp. 630-634., doi:10.1590/abd1806-4841.20175551.

4. Moraites, Eleni, et al. "Incidence and Prevalence of Hyperhidrosis." Dermatologic Clinics, vol. 32, no. 4, 2014, pp. 457-465., doi:10.1016/j.det.2014.06.006.

5. Henning, M. A., et al. "Genetic Disposition to Primary Hyperhidrosis: a Review of Literature." Archives of Dermatological Research, vol. 311, no. 10, 2019, pp. 735-740., doi:10.1007/s00403-019-01966-1.

6. Hashmonai, Moshe, et al. "The Etiology of Primary Hyperhidrosis: A Systematic Review." Clinical Autonomic Research, vol. 27, no. 6, 2017, pp. 379-383., doi:10.1007/s10286-017-0456-0.

7. Gonzalez Fernandez, D. and Perez Oliva, N. "Actualización en hiperhidrosis focal primaria”. Med Cutan Iber Lat Am 2012;40(6):173-180. DOI:10.4464/MD.2012.40.6.5040

8. McConaghy JR, Fosselman D. Hyperhidrosis: Management Options. Am Fam Physician. 2018;97(11):729-734.

9. $\quad$ Swartling, Carl. "Hyperhidrosis - an Unknown Widespread 'Silent' Disorder." Journal of Neurology and Neuromedicine, vol. 1, no. 4, 2016, pp. 25-33., doi:10.29245/2572.942x/2016/4.1037.

10. Gregoriou, Stamatios, et al. "Management Strategies Of Palmar Hyperhidrosis: Challenges And Solutions." Clinical, Cosmetic and Investigational Dermatology, Volume 12, 2019, pp. 733-744., doi:10.2147/ccid. s210973.

11. Nawrocki, Shiri, and Jisun Cha. "The Etiology, Diagnosis, and Management of Hyperhidrosis: A Comprehensive Review." Journal of the American Academy of Dermatology, vol. 81, no. 3, 2019, pp. 669-680., doi:10.1016/j.jaad.2018.11.066. 


\section{CIENCIA\&SALUD}

12. Maillard, H., and P. Dumont. "Hiperhidrosis." EMC - Dermatología, vol. 52, no. 4, 2018, pp. 1-8., doi:10.1016/s1761-2896(18)41449-5.

13. Grabell, Daniel A., and Adelaide A. Hebert. "Current and Emerging Medical Therapies for Primary Hyperhidrosis.” Dermatology and Therapy, vol. 7, no. 1, 2016, pp. 25-36., doi:10.1007/s13555-016-0148-z.

14. Halpert, Evelyne, et al. "Hiperhidrosis Axilar Primaria." Revista De La Asociación Colombiana De Dermatología y Cirugía Dermatológica, vol. 25, no. 4, 2017, pp. 294-302., doi:10.29176/2590843x.303. 